\title{
Multiphysics Modeling of a Wearable Sensor for Sweat Rate Measurements.
}

\author{
Jorge Prada*, Federico Vivaldi*, Andrea Bonini*, Antonio Lanata ${ }^{\dagger}$, Emilio Franchi ${ }^{\ddagger}$ and Fabio Di Francesco* \\ * Department of Chemistry and Industrial Chemistry, University of Pisa, Italy \\ E-mail: jorge.prada@dcci.unipi.it, federicomaria.vivaldi@phd.unipi.it, andrea.bonini@phd.unipi.it, fabio.difrancesco@unipi.it \\ $\dagger$ Department of Information Engineering, University of Florence, Italy \\ E-mail: antonio.lanata@unipi.it \\ ${ }^{\ddagger}$ Research and Development, R.i.CO.srl, Navacchio, Italy \\ E-mail: emilio.franchi@iet.unipi.it
}

\begin{abstract}
Innovative wearable devices are allowing the noninvasive and remote collection of physiological signals from the human body. Sweat analysis has gained remarkable interest for the non-invasive determination of electrolytes and metabolites, particularly in athletes. Monitoring of sweat rate is of interest to support measurements concerning sweat composition and to assess the overall water loss due to physical activity. The final aim is to prevent dehydration and keep an optimal balance of electrolytes, which is important for the safety of athletes and to obtain the best possible performances. We speculate that these devices can also be useful for the protection of workers performing heavy physical tasks. The evaporation of sweat from the skin surface, which is essential for thermal regulation in humans, is largely influenced by ambient factors. Depending on the measurement approach, ambient temperature and humidity as well as air flow do not only affect evaporation but also the measurement of sweat rate. This paper presents a multiphysics model describing the effect of air speed and ambient humidity on an open chamber based sweat rate sensor. Results of simulations will allow to optimize the design of the device so that interferences from ambient conditions are minimized.
\end{abstract}

Index Terms-Sweat rate, open chamber, multiphysics modeling, sensor.

\section{INTRODUCTION}

Technological advances such the miniaturization of electronic devices, the appearance of flexible and stretchable electronics, the reduction of power consumption and the increase of portable computational power have led to significant progresses in the field of wearable sensors. Thanks to these, new wearable medical devices have appeared allowing non-invasive remote monitoring of physiological signals and promising applications for sports and telemedicine. Sweat rate measurement is an application rising noticeable interest within wearable sensor research due to the insightful information that can be retrieved using non-invasive methods. Sweat can be easily an unobtrusively collected and analyzed by wearable sensor systems [1] and smart textiles [2], [3]. Sweat composition and transport of analytes from blood has been studied for long time to identify possible biomarkers offering useful insights on ongoing metabolic processes within the body [4]. The

INAIL is kindly acknowledged for supporting this study within the SenseRisc project (Sviluppo di abiti intelligENti Sensorizzati per prevenzione e mitigazione di RIschi per la SiCurezza dei lavoratori). point measurement of sweat rate is a first step towards the determination of the total body fluid loss, which is important to prevent dehydration in subjects exerting remarkable physical efforts. Knowledge of sweat rate improves the reliability of sweat composition data, as sweat rate affects the concentration of electrolytes in sweat by modifying the efficiency of reabsorption in the duct. Furthermore, a rapid increase of sweat rate has been associated to the transition from aerobic to anaerobic metabolism during physical activity [5]. In addition to the removal of metabolic waste, sweating plays a crucial role in the thermoregulation of the body [6]. The evaporation of sweat (consisting of $98-99 \%$ of water) removes substantial thermal energy from the body, but this phenomenon is largely affected by external factors such as temperature, humidity and air flow. In this paper, a multiphysics model describing the effect of air speed and ambient humidity on an open chamber is presented. Results of simulations can be used to optimize the design of a sweat rate sensor based on an open chamber approach.

\section{A. Measurement of sweat rate}

Sensors to measure sweat rate typically include a chamber for the collection of the humidity released from the skin and one or more humidity sensors [6]. Sweat rate is then calculated either from the rate by which humidity increases over time or from the humidity gradient caused by natural or forced transport within the chamber. The former type of devices are susceptible to saturation of the chamber by the evaporated sweat, so solutions including actuated chamber opening and closing have been proposed [7], [8]. In the later type of devices, the humidity gradient can be produced by a pump-induced air circulation within a closed chamber [9] or by natural ventilation taking place in an open chamber [10]. The simple structure of the open chamber devices makes them wearable and suitable for a continuous monitoring [11], [12]. Nevertheless, their measurements are affected from air flows caused from wind or body movements.

\section{B. Moisture transport model in an open chamber}

The open chamber sweat rate sensor consists of a cylindrical structure lied on the skin in which humidity readings are taken at two different heights. The water vapor arising from either 
insensible or sensible perspiration is channeled through the chamber, so that a humidity gradient is established causing a diffusive flow. Mass diffusion of a substance in a non-uniform mixture follows the first Fick's law:

$$
\vec{j}_{i}=-\rho D_{i} \nabla m_{i}
$$

where $\vec{j}_{i}$ is the mass flow per unit of area and time $\left(\mathrm{kg} /\left(\mathrm{m}^{2} \mathrm{~s}\right)\right), \rho$ is the substance density, $D_{i}$ is the substance diffusivity in the mixture and $m_{i}$ the mass fraction of the substance in the mixture [13]. Adapting Eq. (1) to model the water vapor transport from the skin, and assuming an ideal gas model to describe mixture of water vapor in air, results in:

$$
j_{\text {vapor }}=\frac{D_{\text {vapor }} M_{\mathrm{H}_{2} \mathrm{O}}}{R T}\left(\frac{\Delta P}{\Delta z}\right)
$$

where $M_{\mathrm{H}_{2} \mathrm{O}}$ is the water molar mass $(18.015 \mathrm{~g} / \mathrm{mol}), R$ is the gas constant $(8.314 \mathrm{~J} /(\mathrm{mol} \mathrm{K})), T$ the air temperature in $\mathrm{K}, \Delta P$ the vapor pressure difference $(\mathrm{Pa})$ and $\Delta z$ a reference length $(\mathrm{m})$. In an actual implementation, humidity and temperature sensors are allocated along the water vapor diffusion direction (parallel to the open chamber axis). Humidity and temperature data can be used in Eq. (2) after replacing $\Delta P$ by $r h_{2} P_{s a t, T_{2}}-r h_{1} P_{s a t, T_{1}}$, where $r h$ and $T$ are the measured relative humidity and temperature respectively. $P_{\text {sat }, T}$ is the saturation water vapor pressure at the measured temperature $T$, calculated, for instance, from the Antoine's formula [14].

\section{Previous implementation}

The present multiphysics simulation and analysis of an open chamber device was inspired from a device designed, implemented and tested by Salvo et al. [14]. This device was fitted in a velcro strap to be used for in-vivo tests. Eq. (2) was used to process data from two humidity and temperature sensors and to calculate continuous sweat rate values from 13 athletes performing physical activity [14].

\section{METHODS}

Multiphysics modeling and simulation were performed using COMSOL Multiphysics version 5.4 to verify the impact of different air streams on the moisture transport. The simulated cylindrical chamber is presented in Fig. 1. The open bottom of the structure (under the plate, in z-direction) is in contact with the skin. In order to model the behaviour, the occuring physical phenomena were identified and initial conditions were formulated.

The surrounding air stream was modeled using Turbulent Flow physics in COMSOL. Different air stream approaching angles $\left(0^{\circ},-30^{\circ}\right.$ and $-60^{\circ}$ respect to horizontal line $)$ and velocities (1, 3 and $5 \mathrm{~m} / \mathrm{s})$ were simulated to model some of the possible air dynamics occurring during a walking training session [15], [16]. Additional velocities of 0.01 and $0.10 \mathrm{~m} / \mathrm{s}$ were included to evaluate conditions near to stagnation. Particular interest raised the interaction between the air flow at the entrance of the open chamber and the stagnant air volume within the chamber, that might trigger convective transport of the vapor at the upper tier of the chamber. This interaction

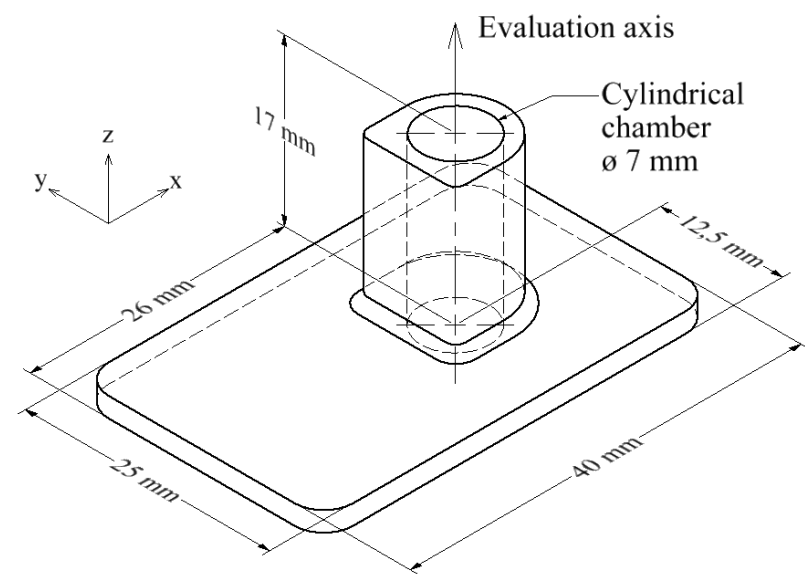

Fig. 1. 3D model of the open chamber. Chamber central axis defined as evaluation axis

was assumed mainly driven by the flow in the vicinity of the fixture surface. For this reason, the Menter's Shear Stress Transport (SST) turbulence model was configured without wall approximation, which required the software to calculate the flow from the surface level by using a very fine mesh in the vicinity of the solid [17].

Although the typical utilization cases do not encompass large temperature gradients between skin and environment, Heat Transfer in Moist Air was configured to account the temperature gradient produced by the heat dissipation from the skin to the surrounding moist air. Skin temperature was assumed at $33^{\circ} \mathrm{C}$, while ambient temperature set at $25^{\circ} \mathrm{C}$ with relative humidity of $70 \%$. Based on these conditions, the first factor at right side of Eq. (2) is calculated as $1.996 \times 10^{-10} \mathrm{~kg} /($ Pa m s $)$. Regarding to moisture transport, the skin surface was modeled as a moist surface with water concentration of $3.26 \mathrm{~mol} / \mathrm{m}^{2}$ [18]. The plastic device fixture was assumed to be thermally insulated in the heat transfer model and its thermal properties were not accounted in the simulation. The skin area was modeled as a boundary latent heat source to account the heat invested in the onset of the water evaporation. Evaporation rate on the skin was assumed at $1.66 \times 10^{-3} \mathrm{~m} / \mathrm{s}$. Moisture transport physics was coupled with the turbulent flow physics to estimate the convective transport of moisture and with the heat transfer physics to account the calculation of evaporation latent heat and saturation pressure at a given temperature distribution.

\section{RESUlts}

Initial results were acquired from a 2-D model representing a vertical cross-section along the chamber axis (plane $\mathrm{xz}$ ). A 2-D representation of the cross-section of the cylindrical chamber from Fig. 1 was arranged inside a frame box of $300 \times 200 \mathrm{~mm}$ representing the surrounding air region. The chamber central axis was set as 'evaluation axis', on which the results from the studies were observed. Top and right sides of the air domain were set as air inlets with $v_{\text {air }}[\hat{x} \cos (\theta)-\hat{z} \sin (\theta)]$ as boundary velocity condition. Ve- 


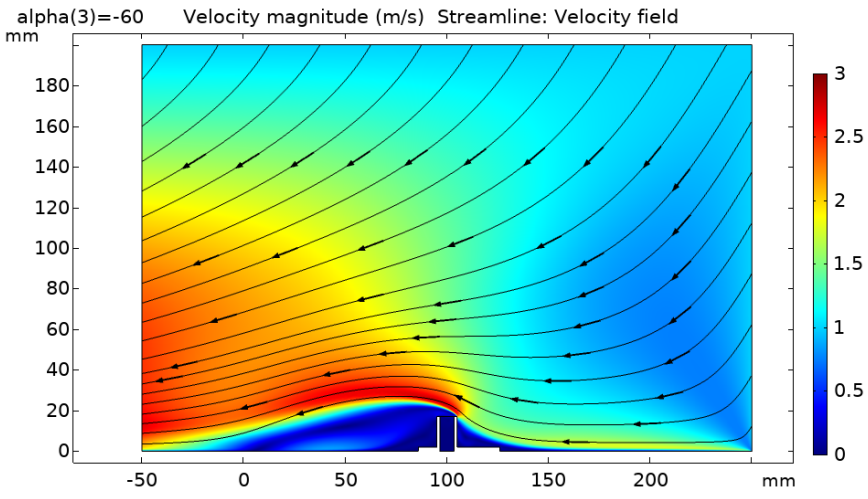

Fig. 2. 2-D distribution of velocity magnitude and streamlines from stationary study. Air flow approaching at $1 \mathrm{~m} / \mathrm{s}$, with an angle of $-60^{\circ}$.

locity magnitude distributions, as shown as example in Fig. 2 for $1 \mathrm{~m} / \mathrm{s},-60^{\circ}$, were obtained after stationary study simulation of turbulent flow in COMSOL. All combinations of angles and air velocities were tested and the corresponding velocity profiles were obtained. On the other hand, a time dependent study analyzed the heat and moisture transfer only within the chamber (setting the outside relative humidity and temperature as boundary conditions at the top of the chamber) to obtain the time distribution of the relative humidity by an ideal undisturbed diffusive transport inside the chamber. Fig. 3 shows the resulting relative humidity at the middle-height point along the evaluation axis over time.

The stabilization time observed in Fig. 3 (20s) was chosen to run the time dependent simulations calculating the heat and moisture transport. Relative humidity and vapor content were observed along the evaluation axis in order to appraise an averaged trend of the phenomena inside the chamber. Results from simulation for the relative humidity, for all velocities are shown in Fig. 4 (with angle of $-60^{\circ}$, as the most disrupting from the studied angles). Additional results for vapor content along the evaluation axis are presented in Fig. 5.

A modified open chamber ( $4 \mathrm{~mm}$ diameter, same height) was modeled and simulated in COMSOL. Same parameters were studied and results for relative humidity along the chamber central axis are presented in Fig. 6 Results from evaluation axis for vapor content are shown in Fig. 7.

\section{DISCUSSION}

Intuitively, one might assume that larger surrounding air speeds and acuter attack angles produce more intense disruption on the diffusive moisture mass transport within the open chamber. In order to validate this assumption, this study aimed to quantify the effect of the transport disruption by evaluating the relative humidity and vapor content along the chamber central axis. Different relative and absolute humidity profiles were obtained at different air flow conditions over a $7 \mathrm{~mm}$ open chamber (Fig. 4 and 5). The larger and larger deviation from the reference cases (low air velocities: 0.01 and $0.10 \mathrm{~m} / \mathrm{s}$ ) suggests that the disruption of moisture transport inside the chamber is strongly correlated to air velocity. Presumably, the

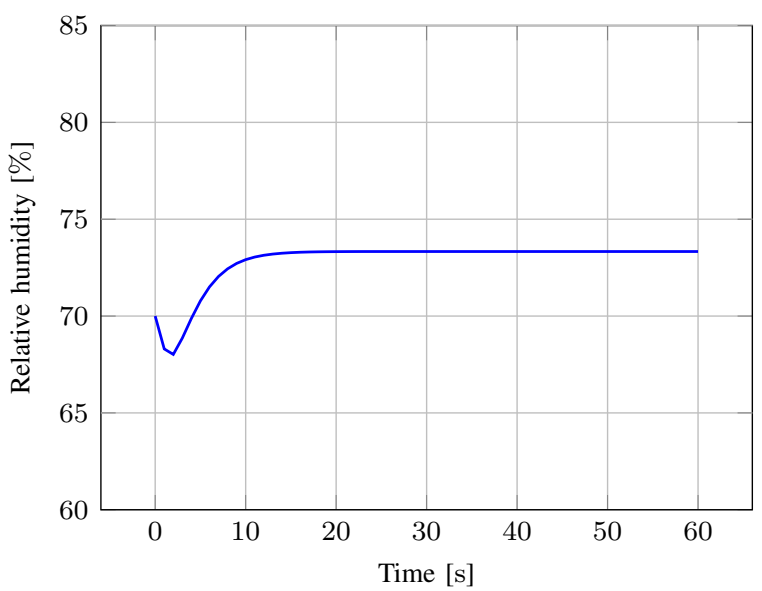

Fig. 3. Time distribution of the relative humidity at the middle-height point, inside the chamber, along the evaluation axis. Diffusive transport modeled inside the chamber and ambient conditions forced at the chamber top (no air flow).

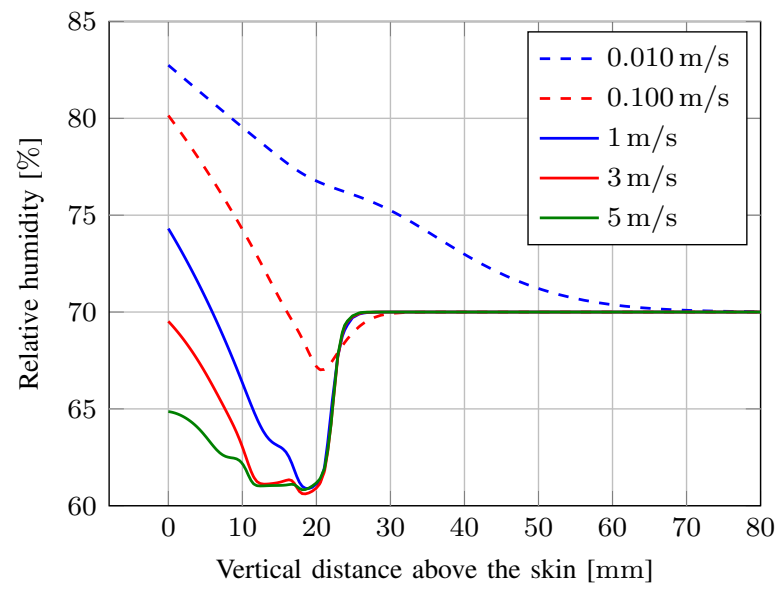

Fig. 4. Relative humidity along the chamber evaluation axis for different velocities $\left(-60^{\circ}\right)$ at $\mathrm{t}=20 \mathrm{~s}$. Chamber diameter was $7 \mathrm{~mm}$.

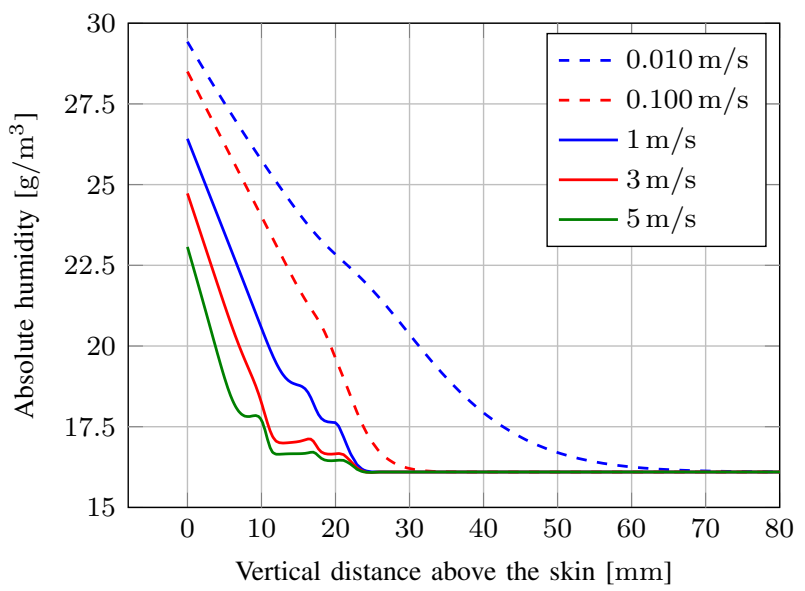

Fig. 5. Vapor content along the chamber evaluation axis for different velocities $\left(-60^{\circ}\right)$ at $\mathrm{t}=20 \mathrm{~s}$. Chamber diameter was $7 \mathrm{~mm}$ 


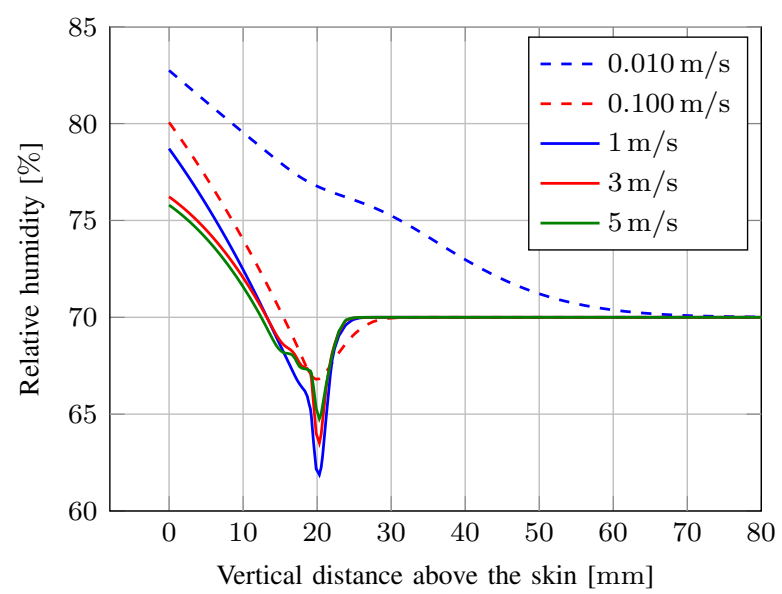

Fig. 6. Relative humidity along the chamber evaluation axis for different velocities $\left(-60^{\circ}\right)$ at $\mathrm{t}=20 \mathrm{~s}$. Chamber diameter was $4 \mathrm{~mm}$.

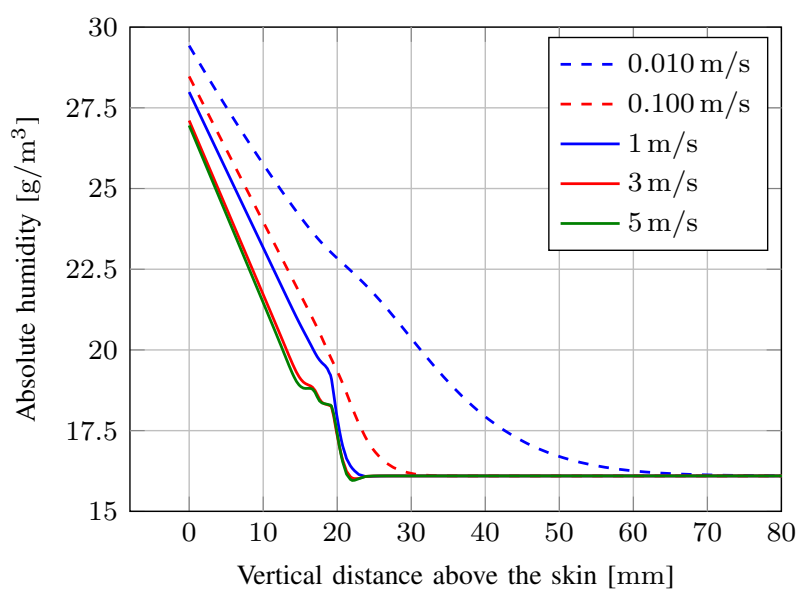

Fig. 7. Absolute humidity along the chamber evaluation axis for different velocities $\left(-60^{\circ}\right)$ at $\mathrm{t}=20 \mathrm{~s}$. Chamber diameter was $4 \mathrm{~mm}$.

onset of air currents and eddies inside the upper section of the chamber diverts the heat and mass transport from the simple 1-D diffusive model (Eq. (2)).

It is worth mentioning that the relative and absolute humidity along the evaluation axis (Fig. 4 and 5) present similar decreasing trends with respect to the distance from the skin, at all air velocities. As expected, the higher the air flow velocity, the lower was relative humidity at the skin level to more effective removal of water vapor. Simulation results do not confirm the presence of a significant convective transport inside the chamber near to the skin, so the enhanced moisture removal might be caused mainly by the diffusive transport of moisture towards the top section of the chamber, where the moisture is depleted by convective transport caused by the air flow. The region where the absolute humidity displays a near to linear behavior in Fig. 5 might then indicate the section of the chamber height in which the diffusive transport of moisture is predominant. The relative humidity behavior, on the other hand, is influenced by the temperature distribution inside the chamber. Higher temperatures close to the skin result in lower relative humidity, explaining the occurrence of values below the ambient relative humidity inside the chamber. Nevertheless, relative humidity is an important variable measured by the moisture sensors and its behavior must be considered when designing the sweat rate sensor.

A remarkable consequence of the relative humidity drop at higher velocities is the reduction of the relative humidity gradient between the skin surface and the chamber top. The measurement of a smaller gradient by an open chamber sweat rate sensor requires humidity sensors with higher precision if the same uncertainty has to be achieved.

Simulations of the narrower chamber (diameter $4 \mathrm{~mm}$ ) suggest that the moisture transport inside the chamber is less affected by the air flow (Fig. 6 and 7). Relative humidity shows larger humidity ranges than in the wider chamber, so that a narrower chamber should improve precision of the sweat rate measurement.

Sweat evaporation in different ambient conditions is a complex phenomenon and numerical results of multiphysics models may divert from experimental values. In our view, the main source of uncertainty in the present case is the assumption of constant boundary conditions, particularly at skin level. Sweating encompasses a progressive increase of the skin temperature and of the amount of liquid sweat available on the skin. The use of simplified 2-D model and the omission of the fixture material are further limitations of this work. A time dependent description of sweating skin and a refinement of the spatial model, as well as the comparison with experimental values, might mitigate present limitations in future works.

Nonetheless, we believe that the present results offer useful insights about the chamber design trade-offs for the later allocation of moisture sensors with a given precision in different use case scenarios. Additional trade-offs concerning manufacturing of the chamber, wearability, compatibility with readout electronics, or even aesthetics, are beyond the scope of this paper.

\section{CONCLUSIONS}

These multiphysics simulations offered a comprehensive overview of the distribution of the evaporated water from a sweating skin within an open chamber. Potential deviations of humidity profiles from the simple 1-D diffusive mass transport model used to calculate sweat rate in open chamber sensors were assessed. Results confirm that air flows affect the humidity gradient within the chamber, but reducing the chamber diameter might help to mitigate such effect. When the open chamber is under the influence of an air flow, higher precision moisture sensors are needed to reduce errors, as large air velocities reduce the differences in humidity values inside the chamber. In this sense, the acknowledge of deviations from the 1-D diffusive model can help to improve the design of the chamber and place the moisture and temperature sensors in an optimal location. Furthermore, this information can be used to correct the sensor calibration curve and improve accuracy. 
Despite the elaboration of our multiphysics modeling for the sweat evaporation phenomena, the numerical results may divert significantly from experimental values, although we do not expect that to substantially divert the here presented conclusions. Limitations of the present simulations are the use of a simplified 2-D model, the omission of the fixture material in the heat transport model and the assumption of constant boundary conditions. A refinement of the spatial model, a more detailed skin and ambient description and their correction with experimental values might mitigate the present limitations in future related works.

\section{REFERENCES}

[1] S. Coyle, K. Lau, N. Moyna, D. O'Gorman, D. Diamond, F. D. Francesco, D. Costanzo, P. Salvo, M. G. Trivella, D. E. D. Rossi, N. Taccini, R. Paradiso, J. Porchet, A. Ridolfi, J. Luprano, C. Chuzel, T. Lanier, F. Revol-Cavalier, S. Schoumacker, V. Mourier, I. Chartier, R. Convert, H. De-Moncuit, and C. Bini, "BIOTEX-Biosensing Textiles for Personalised Healthcare Management," IEEE Transactions on Information Technology in Biomedicine, vol. 14, no. 2, pp. 364-370, 2010.

[2] C. Legner, U. Kalwa, V. Patel, A. Chesmore, and S. Pandey, "Sweat sensing in the smart wearables era: Towards integrative, multifunctional and body-compliant perspiration analysis," Sensors and Actuators A: Physical, vol. 296, pp. 200-221, 2019. [Online]. Available: http://www.sciencedirect.com/science/article/pii/S0924424719307915

[3] C. Cochrane, C. Hertleer, and A. Schwarz-Pfeiffer, "2 - Smart textiles in health: An overview," in Smart Textiles and Their Applications, V. B. T. S. T. Koncar and their Applications, Eds. Oxford: Woodhead Publishing, 2016, ch. 2, pp. 9-32. [Online]. Available: http://www.sciencedirect.com/science/article/pii/B9780081005743000023

[4] A. J. Bandodkar, W. J. Jeang, R. Ghaffari, and J. A. Rogers, "Wearable Sensors for Biochemical Sweat Analysis," Annual Review of Analytical Chemistry, vol. 12, no. 1, pp. 1-22, jun 2019. [Online]. Available: https://doi.org/10.1146/annurev-anchem-061318-114910

[5] A. Pingitore, P. Salvo, F. Mastorci, G. Catapano, L. Sordi, P. Piaggi, and F. Di Francesco, "Sweat Rate Monitoring During Maximal Exercise in Healthy Soccer Players: A Close Relationship with Anaerobic Threshold," Annals of Sport Medicine and Research, vol. 2, no. 8, p. 1047, 2015.

[6] F. Di Francesco, S. Tabucchi, L. Murgia, and M. Onor, "L'analisi della composizione del sudore come strumento d'indagine medica," in Sistemi indossabili per la salute e la protezione dell'uomo. Pàtron, 2008, pp. 259-289.

[7] J. K. Sim and Y. Cho, "Portable active sensors for human sweat rate monitoring," in 2015 IEEE SENSORS, 2015, pp. 1-4.

[8] J. K. Sim and Y.-H. Cho, "Portable sweat rate sensors integrated with air ventilation actuators," Sensors and Actuators B: Chemical, vol. 234, pp. 176-183, 2016. [Online]. Available: http://www.sciencedirect.com/science/article/pii/S0925400516306190

[9] Y. Tsai and C. Chen, "Development and testing of a perspiration measuring system," Medical Engineering \& Physics, vol. 32, no. 4, pp. 356-362, 2010. [Online]. Available: http://www.sciencedirect.com/science/article/pii/S1350453310000093

[10] G. E. Nilsson, "Measurement of water exchange through skin," Medical and Biological Engineering and Computing, vol. 15, no. 3, pp. 209-218, 1977. [Online]. Available: https://doi.org/10.1007/BF02441040

[11] S. Coyle, D. Morris, K. Lau, D. Diamond, N. Taccini, D. Costanzo, P. Salvo, F. D. Francesco, M. G. Trivella, J. Porchet, and J. Luprano, "Textile sensors to measure sweat $\mathrm{pH}$ and sweat-rate during exercise," in 2009 3rd International Conference on Pervasive Computing Technologies for Healthcare, 2009, pp. 1-6.

[12] P. Salvo, F. D. Francesco, D. Costanzo, C. Ferrari, M. G. Trivella, and D. D. Rossi, "A Wearable Sensor for Measuring Sweat Rate," IEEE Sensors Journal, vol. 10, no. 10, pp. 1557-1558, 2010.

[13] J. H. Lienhard, A Heat Transfer Textbook. Phlogiston Press, 2003. [Online]. Available: https://books.google.de/books?id=GBImuwEACAAJ
[14] P. Salvo, A. Pingitore, A. Barbini, and F. Di Francesco, "A wearable sweat rate sensor to monitor the athletes' performance during training," Science \& Sports, vol. 33, no. 2, pp. e51-e58, 2018. [Online]. Available: http://www.sciencedirect.com/science/article/pii/S076515971730182X

[15] L. Pugh, "The influence of wind resistance in running and walking and the mechanical efficiency of work against horizontal or vertical forces," The Journal of Physiology, vol. 213, no. 2, pp. 255-276, mar 1971. [Online]. Available: https://doi.org/10.1113/jphysiol.1971.sp009381

[16] T. F. Novacheck, "The biomechanics of running," Gait \& Posture, vol. 7, no. 1, pp. 77-95, 1998. [Online]. Available: http://www.sciencedirect.com/science/article/pii/S0966636297000386

[17] W. Frei, "Which Turbulence Model Should I Choose for My CFD Application?" 2017. [Online]. Available: https://www.comsol.com/blogs/which-turbulence-model-shouldchoose-cfd-application/

[18] E. Jonathan, "In vivo sweat film layer thickness measured with Fourierdomain optical coherence tomography (FD-OCT)," Optics and Lasers in Engineering, vol. 46, no. 6, pp. 424-427, 2008. [Online]. Available: http://www.sciencedirect.com/science/article/pii/S0143816608000183 